\title{
Supplementary Information: Revealing Charge-Transfer Dynamics at Electrified Sulfur Cathodes Using Constrained Density Functional Theory
}

Yierpan Aierken ${ }^{\dagger}$ Ankit Agrawal, ${ }^{\dagger}$ Meiling Sun, ${ }^{\ddagger}$ Marko Melander, ${ }^{\top}$ Ethan J. Crumlin, ${ }^{\ddagger}, \S$ Brett A. Helms, ${ }^{\dagger}, \|$ and David Prendergast ${ }^{*, \dagger}$

$\dagger$ The Molecular Foundry, Lawrence Berkeley National Laboratory, 1 Cyclotron Road, Berkeley, CA 94720, USA

$\ddagger$ Advanced Light Source, Lawrence Berkeley National Laboratory, 1 Cyclotron Road, Berkeley, CA 94720, USA

\Nanoscience Center, Department of Chemistry, University of Jyväskylä, P.O. Box 35 (YN) FI-40014, FinLand

$\S$ Chemical Sciences Division, Lawrence Berkeley National Laboratory, 1 Cyclotron Road, Berkeley, CA 94720, USA

||Materials Sciences Division, Lawrence Berkeley National Laboratory, 1 Cyclotron Road, Berkeley, CA 94720, USA

E-mail: dpendergast@lbl.gov 


\section{Computational Details}

All of the density functional theory (DFT) computations in this work employed the GPAW code. ${ }^{1 / 2}$ The atomic structural models mostly comprised a slab model of an open surface with a 98-atom graphene sheet, and one or three molecular layers of $\alpha-\mathrm{S}_{8}$ (extracted from the rhombohedral unit cell) of 64 or 192 atoms, respectively. The atomic structure optimization was performed in the linear combination of atomic orbitals (LCAO) mode, ${ }^{3}$ with the default double-zeta polarized basis set. The rest of the calculations, such as total energy, density of states, and RT-TDDFT were performed in the finite-difference (FD) mode, where the wave functions are expanded on a real space grid. A grid spacing of $0.18 \AA$ was chosen for accurate total energies. We used the Perdew-BurkeErnzerhof (PBE) exchange-correlation functional ${ }^{4}$ for our spin-polarized calculations, with dipole-corrections applied perpendicular to the slab.

The constrained-DFT (CDFT) ${ }^{5}$ implementation in GPAW ${ }^{6}$ is used to constrain the electronic charge on graphene. The constrained volume is defined by the Hirschfeld partitioning scheme, and, in our example, includes all the $\mathrm{C}$ atoms in graphene. The constrained charge was initially set to be $-1 e$, but was later optimized to balance the net charge of Li. Due to orbital hybridization around the Li ion, its net charge can vary from the nominal +1 for an isolated ion. However, the overall charge of the entire supercell is always zero, unless specified otherwise.

Our real-time time-dependent DFT (RTTDDFT) simulations were initialized using the atomic and electronic structure resulting from 2 ps of molecular dynamics (MD) thermalization applied together with the aforementioned electronic constraint (holding $-1 e$ on the graphene) using CDFT. The MD time step was 1 fs. We sampled the NVT ensemble at $300 \mathrm{~K}$. The resulting atomic structure, together with the electronic orbitals, are then supplied to the RT-TDDFT algorithm and evolved according to Ehrenfest dynamics (ED). ${ }^{7}$ This allows the electronic structure to evolve free of the elec- tronic constraint imposed by CDFT. The time step employed in the ED here was 20 attoseconds.

All visualizations were done in OVITO. 8

\section{The Neutral $\mathrm{LiS}_{8}$ Complex}

In the main manuscript, we have seen that significant increases in a S-S bond length in a given $\mathrm{S}_{8}$ molecule are due to gaining one electron (reduction). Figure S1 provides an isolated molecular analog of this expected phenomenon. The neutral $\mathrm{LiS}_{8}$ complex, calculated using standard, unconstrained DFT, results in complete electron transfer from the $\mathrm{Li}$ ion to the nearby sulfur atoms, producing what could be viewed as an $\mathrm{S}_{8}^{-\cdot}$ radical, with the additional electron localized in the most elongated S-S bond (as shown in green). This could be viewed as the first step in forming a linear polysulfide during the Li-S discharge process.

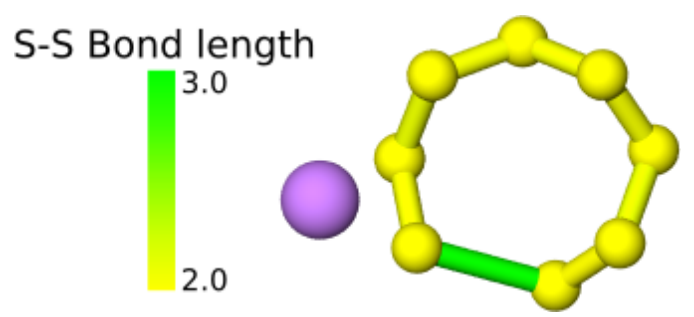

Figure S1: The isolated, neutral $\mathrm{LiS}_{8}$ complex, exhibiting one elongated S-S bond due to electron transfer from Li to the $\mathrm{S}_{8}$ molecule, analogous to the charge localization in extended systems. Atomic colors: Li - purple; S - yellow. $\mathrm{S}-\mathrm{S}$ bond length colored according to the scale bar, in the range $2-3 \AA$

\section{Relaxed Structures for Var- ious Li Ion Positions}

In the main manuscript's discussion of Figure 5 , we provide analysis of the total energy of the graphene-sulfur-Li-ion system for a sequence of Li-ion positions inside the sulfur, defining a path from the open surface to the interface with graphene. In Figure S2, we provide details of the S-S bond lengths within the sulfur slab as a 
CDFT

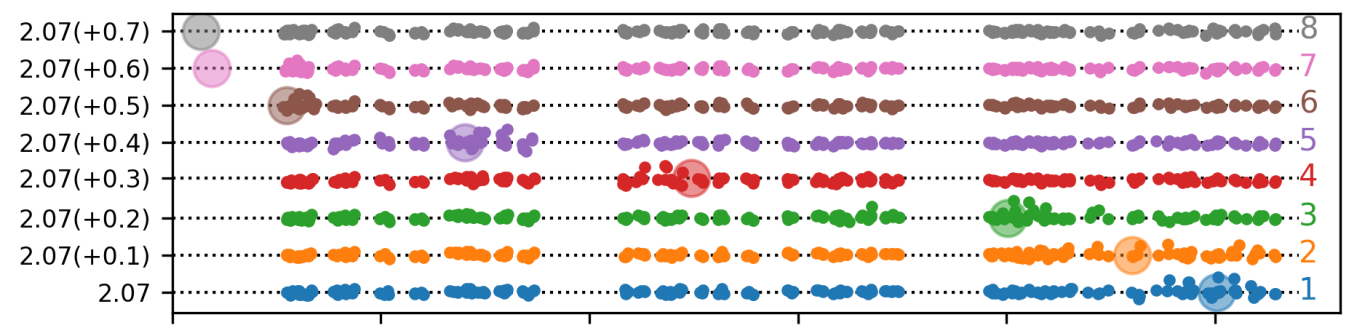

DFT

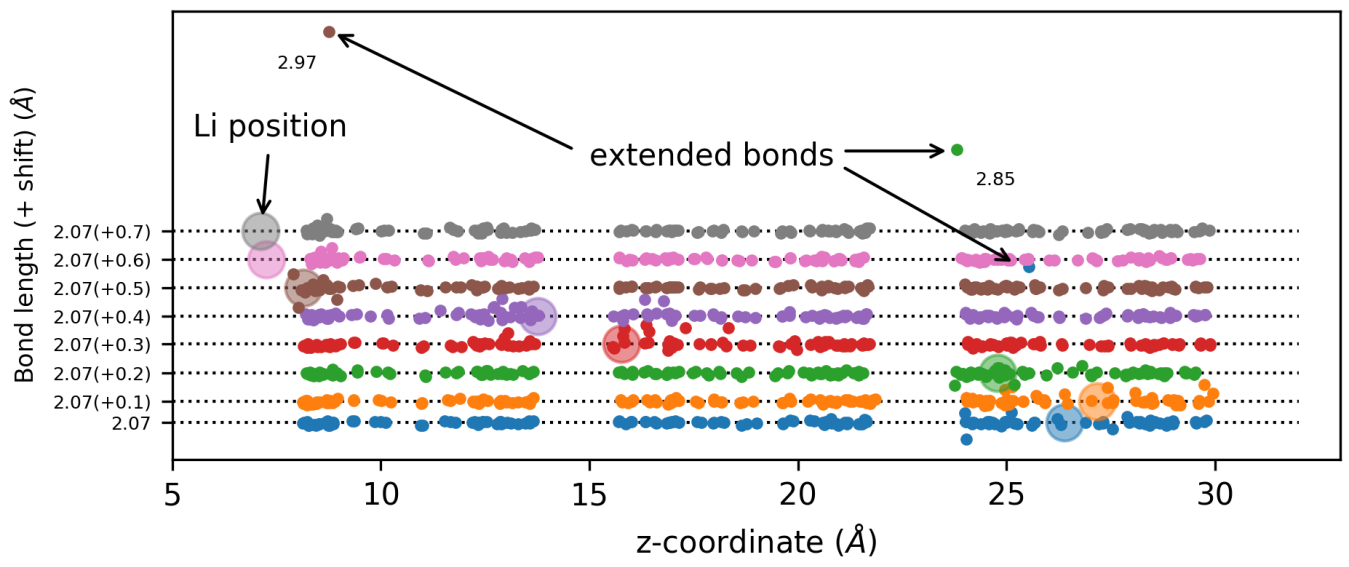

Figure S2: A comparison of S-S bond lengths as a function of position perpendicular to the graphene sheet for a range of $\mathrm{Li}$ ion positions (indicated by larger colored circles). Data for each successive Li-ion position are offset vertically for clarity. Top: Constrained DFT. Bottom: Unconstrained DFT.

(a) structure 1

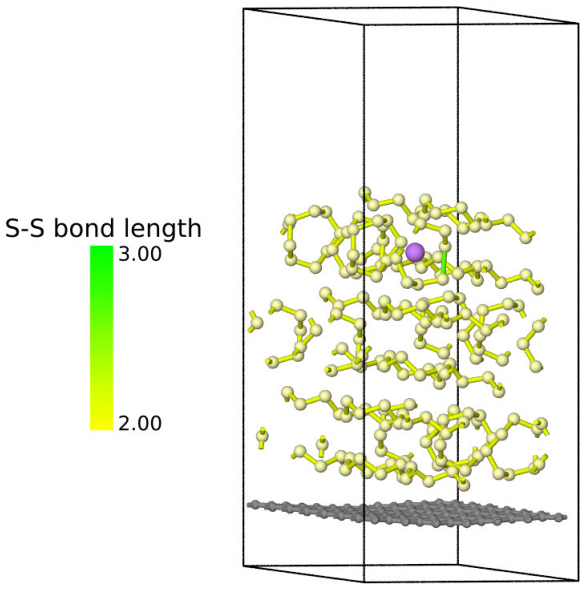

(b) structure 3

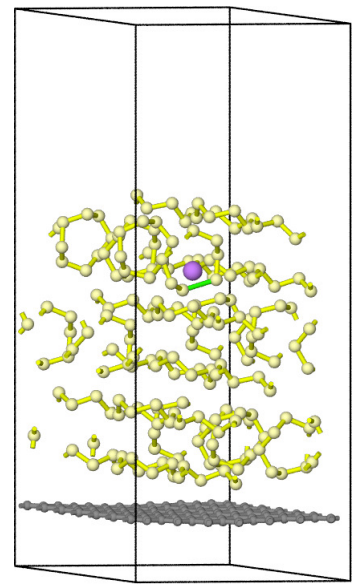

(c) structure 6

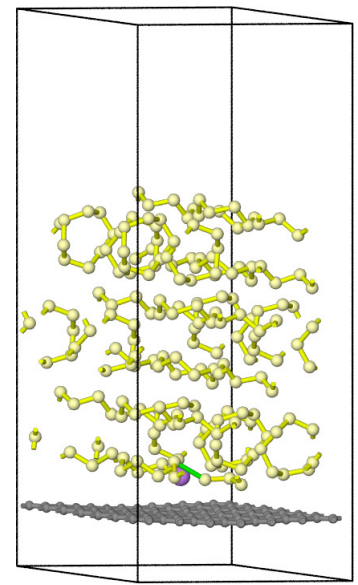

Figure S3: Relaxed, unconstrained DFT structures of 1 (a), 3 (b) and 6 (c) from Figure S2 indicating the clearly extended S-S bond close to the Li-ion due to electronic reduction. 

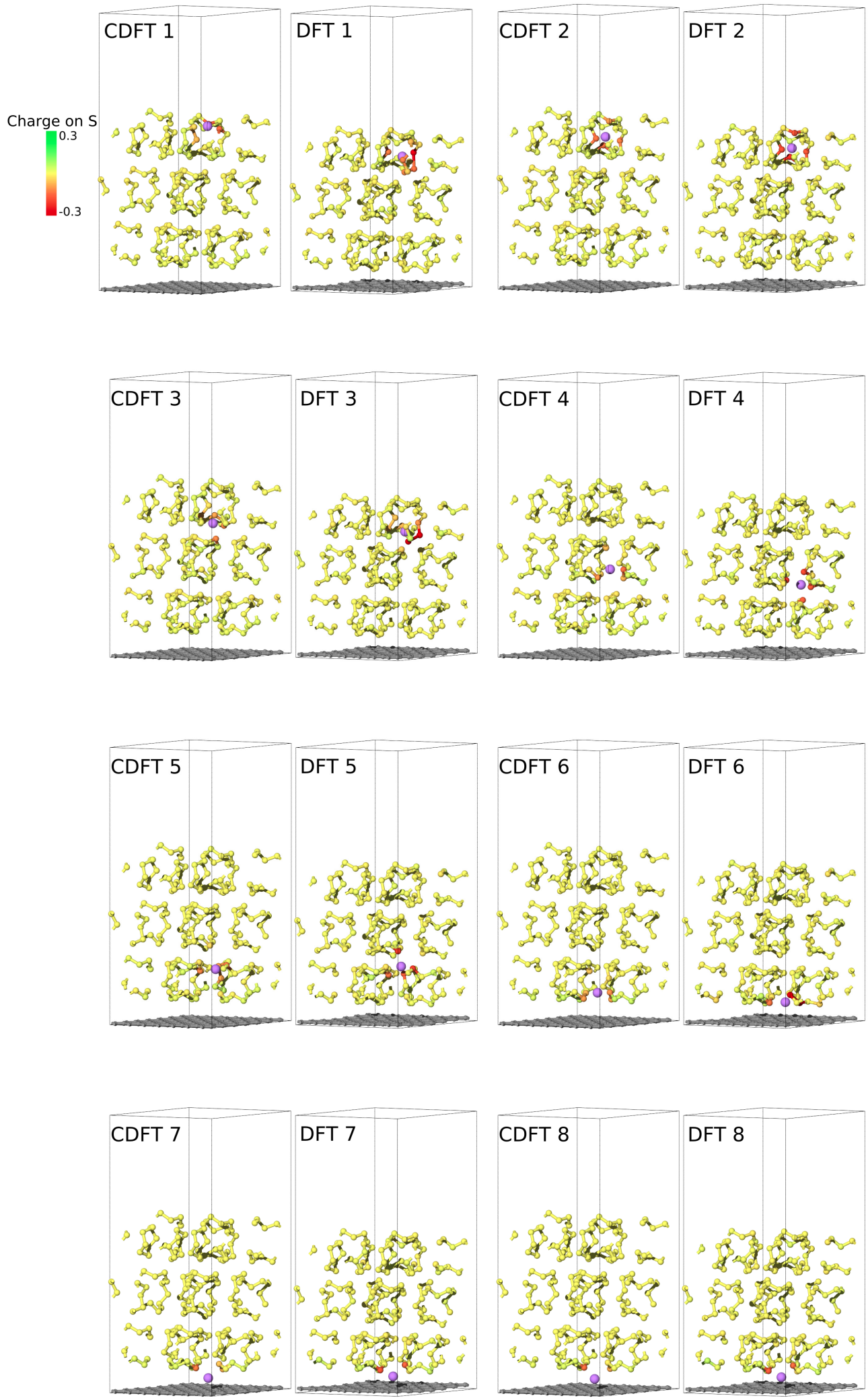

Figure S4: All structures resulting from structural relaxation for various Li ion positions computed using constrained and unconstrained DFT (side by side), indicating by color the net charge on S atoms. 
function of their $z$ coordinate (the graphene is located closest to zero) for each position of the Li-ion. The mean bond length is $2.07 \AA$, and the plot indicates how much the bond lengths deviate from the mean (with a vertical offset to reveal the data from each Li-ion position). Comparing the results from constrained and unconstrained DFT calculations, it is clear that unconstrained calculations display larger deviations in the $\mathrm{S}-\mathrm{S}$ bond lengths in those regions around the Li-ion. In some specific cases, the bond could be considered as broken (extending almost to $3 \AA$ ) due to complete electron transfer, analogous to Fig. S1. The atomic structure of these configurations is shown in Figure S3, with the extended bonds clearly colored in green. The most extended bonds are always in the vicinity of the Li-ion. For completeness, all of the relaxed structures from these calculations are shown in Figure S4 with atoms colored according to their net charge. The main observation is that unconstrained DFT calculations typically lead to significant electron transfer to sulfur atoms locally coordinating the Li ion, and, in some cases, exhibit bond breaking or polysulfide radical formation. However, constrained DFT calculations prevent such charge transfer from occurring and, instead, reveal the structural and charge rearrangements in the sulfur due to electrostatic polarization.

\section{References}

(1) Mortensen, J. J.; Hansen, L. B.; Jacobsen, K. W. Real-space grid implementation of the projector augmented wave method. Physical Review B 2005, 71.

(2) Enkovaara, J.; Rostgaard, C.; Mortensen, J. J.; Chen, J.; Dułak, M.; Ferrighi, L.; Gavnholt, J.; Glinsvad, C.; Haikola, V.; Hansen, H. A. et al. Electronic structure calculations with GPAW: a real-space implementation of the projector augmented-wave method. Journal of Physics: Condensed Matter 2010, 22, 253202.

(3) Larsen, A. H.; Vanin, M.; Mortensen, J. J.;
Thygesen, K. S.; Jacobsen, K. W. Localized atomic basis set in the projector augmented wave method. Physical Review B 2009, 80.

(4) Perdew, J. P.; Burke, K.; Ernzerhof, M. Generalized Gradient Approximation Made Simple. Physical Review Letters 1996, 77, 3865-3868.

(5) Kaduk, B.; Kowalczyk, T.; Voorhis, T. V. Constrained Density Functional Theory. Chemical Reviews 2011, 112, 321-370.

(6) Melander, M.; Jónsson, E. O.; Mortensen, J. J.; Vegge, T.; Lastra, J. M. G. Implementation of Constrained DFT for Computing Charge Transfer Rates within the Projector Augmented Wave Method. Journal of Chemical Theory and Computation 2016, 12, 5367-5378.

(7) Ojanperä, A.; Havu, V.; Lehtovaara, L.; Puska, M. Nonadiabatic Ehrenfest molecular dynamics within the projector augmented-wave method. The Journal of Chemical Physics 2012, 136, 144103.

(8) Stukowski, A. Visualization and analysis of atomistic simulation data with OVITOthe Open Visualization Tool. Modelling and Simulation in Materials Science and Engineering 2010, 18. 